\title{
Note on symplectic SVD-like decomposition
}

\author{
Agoujil Said ${ }^{1}$ and Bentbib Abdeslem Hafid ${ }^{2}$ \\ ${ }^{1}$ Department of Computer Science, Faculty of Science and Technology Errachidia, Morocco \\ 2 Department of Computer Science, Faculty of Science and Technology Marrakech, Morocco
}

Received: 10 October 2015, Revised: 10 October 2015, Accepted: 6 January 2016

Published online: 21 February 2016.

\begin{abstract}
The aim of this study was to introduce a constructive method to compute a symplectic singular value decomposition (SVDlike decomposition) of a $2 n$-by- $m$ rectangular real matrix $A$, based on symplectic reflectors. This approach used a canonical Schur form of skew-symmetric matrix and it allowed us to compute eigenvalues for the structured matrices as Hamiltonian matrix $J A A^{T}$.
\end{abstract}

Keywords: Singular value decomposition (SVD), Hamiltonian matrix, skew-symmetric matrix, skew-Hamiltonian matrix, symplectic matrix, Schur form, symplectic reflector.

\section{Introduction}

Singular Value Decomposition (SVD) has been used in many fields of scientific computing such as data compression, signal processing, automatic control working on applied linear algebra, signal and image processing [13, 14]. An example is about the eigenvalue problem of the matrix

$$
F=\left[\begin{array}{cc}
-C & -G \\
I & 0
\end{array}\right]=\left[\begin{array}{cc}
-C & -I 0 \\
I & 0
\end{array}\right]\left[\begin{array}{cc}
I & 0 \\
0 & G
\end{array}\right]
$$

which is related to the gyroscopic system $[10,11,12]$

$$
q "+C q^{\prime}+G q=0 ; q(0)=q_{0} ; q^{\prime}(0)=q_{1}
$$

A matrix $G \in \mathbb{R}^{m \times m}$ is symmetric and positive semi-definite it has a full rank factorization $G=L L^{T}$. And $C \in \mathbb{R}^{m \times m}$ is skew-symmetric. By using the equality

$$
\left[\begin{array}{cc}
-C & -I \\
I & 0
\end{array}\right]=\left[\begin{array}{cc}
-\frac{1}{2} C & I \\
I & 0
\end{array}\right] J\left[\begin{array}{rr}
\frac{1}{2} C & I \\
I & 0
\end{array}\right]
$$

where $J=\left(\begin{array}{cc}0 & I_{n} \\ -I_{n} & 0\end{array}\right), I_{n}$ denotes the $n \times n$ identity matrix, $F$ is similar to the Hamiltonian matrix

$$
J\left[\begin{array}{cc}
\frac{1}{2} C & I \\
I & 0
\end{array}\right]\left[\begin{array}{cc}
I & 0 \\
0 & L L^{T}
\end{array}\right]=J\left[\begin{array}{cc}
-\frac{1}{2} C & I \\
L^{T} & 0
\end{array}\right]^{T}\left[\begin{array}{cc}
-\frac{1}{2} C & I \\
L^{T} & 0
\end{array}\right]
$$


Therefore the eigenvalue problem of $F$ can be solved by computing the $S V D$-like decomposition of $\left(\begin{array}{cc}-\frac{1}{2} C & I \\ L^{T} & 0\end{array}\right)$.

This paper makes a constructive and significant contribution to this area of research for computing a symplectic SVD-like decomposition of $2 n$-by- $m$ real matrix based on a canonical Schur form of skew-symmetric matrix [16, 17] and by the use of symplectic reflectors [1,2,3]. A method for computing an SVD-like decomposition was given by Hongguo $\mathrm{Xu}[16,17]$ of a $n$-by- $2 m$ real matrix.

Symplectic SVD-like decomposition is effective for computing the structured canonical form of the Hamiltonian matrix $A^{J} A$. Most eigenvalue problems that arise in practice are known to be structured. Therefore, preserving the structure can help preserve physically relevant symmetries in the eigenvalues of the matrix and may improve the accuracy and efficiency of eigenvalue computation. Hamiltonian and skew-Hamiltonian eigenvalue problems arise from a number of applications, particularly in systems and control theory $[7,12,15]$.

The paper is organized as follows: section 2 introduces some notation and some basic result; a symplectic SVD-like decomposition is proposed in section 3; and section 4 we give a numerical results to demonstrate the effectiveness of the proposed algorithm.

\section{Terminology, notation and some basic facts}

In this section, we recall some notations and necessary tools which will be used throughout this paper. The $J$-transpose of any $2 n$-by- $2 p$ matrix $M$ is defined by $M^{J}=J_{2 p}^{T} M^{T} J_{2 n} \in \mathbb{R}^{2 p \times 2 n}$ where $J_{2 n}=\left(\begin{array}{cc}O_{n} & I_{n} \\ -I_{n} & O_{n}\end{array}\right)$, with $I_{n}$ and $O_{n}$ are the $n \times n$ identity and zero matrix respectively. A Hamiltonian matrix $M \in \mathbb{R}^{2 n \times 2 n}$ has the explicit block structure $M=\left(\begin{array}{c}A \\ R \\ G-A^{T}\end{array}\right)$, where $A, G, R$ are real $n \times n$ matrices and $G=G^{T}, R=R^{T}$. By straightforward algebraic manipulation, we can show that a Hamiltonian matrix $M$ is equivalently defined by the property $M^{J}=-M$. Likewise, a matrix $M$ is skew-Hamiltonian if and only if $M^{J}=-M$, it has the explicit block structure $W=\left(\begin{array}{cc}A & R \\ G & A^{T}\end{array}\right)$, where $A, G, R$ are real $n \times n$ matrices and $G=-G^{T}, R=-R^{T}$. Any matrix $S \in \mathbb{R}^{2 n \times 2 p}$ that satisfies this property $S^{T} J_{2 n} S=J_{2 p}\left(S^{J} S=I_{2 p}\right)$ is called symplectic matrix. This property is also called $J$-orthogonality. The symplectic similarity transformations preserve Hamiltonian and skew-Hamiltonian structures.

Proposition 1. An augmented matrix

$$
S=\left(\begin{array}{cccc}
I & 0 & 0 & 0 \\
0 & P_{11} & 0 & P_{12} \\
0 & 0 & I & 0 \\
0 & P_{21} & 0 & P_{22}
\end{array}\right)
$$

is symplectic if and only if $P=\left(\begin{array}{cc}P_{11} & P_{12} \\ P_{21} & P_{22}\end{array}\right)$ is also symplectic.

Setting $E_{i}=\left[e_{i} e_{n+i}\right] \in \mathbb{R}^{2 n \times 2}$ for $i=1, \cdots, n$, we obtain

$$
E_{i}^{J}=E_{i}^{T} \text { and } E_{i}^{J} E_{j}=\delta_{i j} I_{2}
$$

where

$$
\delta_{i j}= \begin{cases}1 & \text { if } i=j \\ 0 & \text { if } i \neq j\end{cases}
$$


Proposition 2. Let $U=\left[\begin{array}{ll}u_{1} & u_{2}\end{array}\right]$ be a $2 n$-by-2 real matrix, where $u_{1}=\sum_{i=1}^{2 n} u_{i}^{(1)} e_{i}$ and $u_{2}=\sum_{j=1}^{2 n} u_{j}^{(2)} e_{j}$. Then, $U$ is written uniquely as linear combination of $\left(E_{i}\right)_{1 \leq i \leq n}$ on the ring $\mathbb{R}^{2 \times 2}$.

$$
U=\sum_{i=1}^{n} E_{i} M_{i} \text { where } M_{i}=\left(\begin{array}{cc}
u_{i}^{(1)} & u_{i}^{(2)} \\
u_{n+i}^{(1)} & u_{n+i}^{(2)}
\end{array}\right)
$$

Proposition 3. Let $M$ be a $2 n$-by-2n real matrix. Then, $M$ is expressed uniquely as $M=\sum_{i=1}^{n} \sum_{j=1}^{n} E_{i} M_{i j} E_{j}^{T}$ where $M_{i j} \in$ $\mathbb{R}^{2 s \times 2 s}$ is given by,

$$
\left(\begin{array}{l|l}
m_{i, j} & m_{i, n+j} \\
\hline m_{n+i, j} & m_{n+i, n+j}
\end{array}\right) .
$$

Proposition 4. With the notations of the previous proposition, a matrix $M \in \mathbb{R}^{2 n \times 2 n}$ is Hamiltonian (skew-Hamiltonian) if $M_{i j}^{J}=-M_{j i}\left(M_{i j}^{J}=M_{j i}\right)$.

Proof. The result is obvious, as $M^{J}=\sum_{i=1}^{n} \sum_{j=1}^{n} E_{i} M_{j i}^{J} E_{j}^{T}$ and $M^{J}=-M$.

Definition 1. A matrix $M=\sum_{i=1}^{n} \sum_{j=1}^{n} E_{i} M_{i j} E_{j}^{T} \in \mathbb{R}^{2 n \times 2 n}$ is called in upper J-bidiagonal form if $M_{i j}=0_{2}$ for $j \notin\{i, i+1\}$ and, in addition, $M_{i i}$ and $M_{i i+1}$ are diagonal.

\subsection{Symplectic reflectors}

The symplectic reflector [2,3] in $\mathbb{R}^{2 n \times 2}$ is defined in parallel with elementary reflectors.

Proposition 5. [3] Let $U$ and $V$ be two $2 n$-by-2 real matrices that satisfy $U^{J} U=V^{J} V=I_{2}$. If the 2-by-2 matrix $C=$ $I_{2}+V^{J} U$ is nonsingular, the transformation $S=(U+V) C^{-1}(U+V)^{J}-I_{2 n}$ is symplectic and takes $U$ to $V$. This is called a symplectic reflector. Additionally, if $U^{J}=U^{T}$ and $V^{J}=V^{T}$, then $S$ is orthogonal and symplectic.

Remark. The proposition above remains true only if $U^{J} U=V^{J} V$. In this case, $C=U^{J} U+V^{J} U$.

Lemma 1. Let $U=\left[\begin{array}{ll}u_{1} & u_{2}\end{array}\right] \in \mathbb{R}^{2 n \times 2}$ be a non-isotropic matrix $\left(U^{J} U \neq 0_{2}\right)$ and $V=U q(U)^{-1}$ its normalized matrix. Then, there is a symplectic reflector $S$ takes $V$ to $E_{1}$ and therefore $U$ to $E_{1} q(U)$, which in turn takes the following form:

$$
S U=\left(\begin{array}{cc}
* & 0 \\
0 & 0 \\
\vdots & \vdots \\
0 & 0 \\
0 & * \\
0 & 0 \\
\vdots & \vdots \\
0 & 0
\end{array}\right) \swarrow n+1
$$

where

$$
\begin{gathered}
q(U)=\left\{\begin{array}{cc}
\sqrt{\alpha} I_{2}, & \text { if } \alpha>0 \\
\sqrt{-\alpha}\left(\begin{array}{cc}
1 & 0 \\
0 & -1
\end{array}\right), & \text { if } \alpha<0
\end{array}\right. \\
\alpha=u_{1}^{H} J u_{2} .
\end{gathered}
$$


Remark.Using symplectic reflectors with a matrix $A \in \mathbb{R}^{2 n \times 2 n}$, we obtain the factorization $A=S R$, where $S \in \mathbb{R}^{2 n \times 2 n}$ is symplectic and $R=\left(\begin{array}{ll}R_{11} & R_{12} \\ R_{21} & R_{22}\end{array}\right) \in \mathbb{R}^{2 n \times 2 n} . R$ is $J$-triangular and, in addition, $R_{12}$ is a strictly $n$-by- $n$ upper triangular matrix. $R$ is as follows:
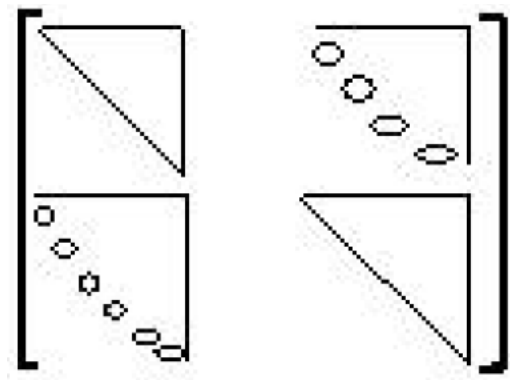

We discuss below some useful properties of symplectic reflectors.

Proposition 6. Let $S$ be a $2 n$-by-2n real symplectic matrix. There is then a sequence of symplectic reflectors $S_{1}, S_{2}, \cdots, S_{n}$, such that $S=S_{1} S_{2} \cdots S_{n}$.

Proof. Step 1: Set $U_{1}=\left[q_{1}, q_{n+1}\right] \in \mathbb{R}^{2 n \times 2}$. As $S$ is symplectic, then $U_{1}^{J} U_{1}=I_{2}$. Then, the symplectic reflector $P_{1}=$ $\left(U_{1}+E_{1}\right)\left(I_{2}+E_{1}^{J} U_{1}\right)^{-1}\left(U_{1}+E_{1}\right)^{J}-I_{2 n}$ verifies $P_{1} U_{1}=E_{1}$. The $(n+1)^{t h}$-component of both $\left(P_{1} q_{k}\right)$ and $\left(P_{1} q_{n+k}\right)$ is equal to zero for $k=2,3, \ldots n$. On the one hand, $\left(P_{1} q_{1}\right)^{T} J\left(P_{1} q_{k}\right)=q_{1}^{T} J q_{k}=0$, and on the other hand, $\left(P_{1} q_{1}\right)^{T} J\left(P_{1} q_{k}\right)=$ $e_{1}^{T} J\left(P_{1} q_{k}\right)=e_{n+1}^{T}\left(P_{1} q_{k}\right)$ is simply the $(n+1)^{t h}$-component of $\left(P_{1} q_{k}\right)$. Likewise, the first component of both $\left(P_{1} q_{k}\right)$ and $\left(P_{1} q_{n+k}\right)$ disppears. Finally, we obtain

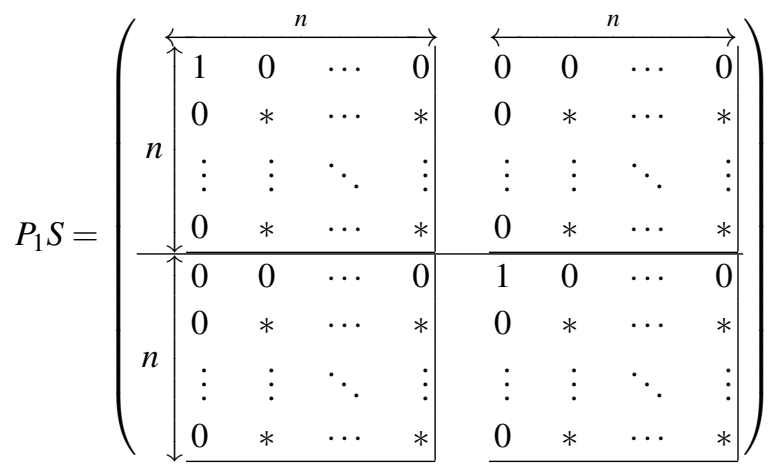

Thereafter, we continue to update the value of $q_{i}: q_{i} \longleftarrow P_{1} q_{i}$ by varying $i$ from 1 to $2 n$. Note that now we have $q_{1}=e_{1}$ and $q_{n+1}=e_{n+1}$.

Step 2: Set $U_{1}=\left[q_{1}, q_{n+1}\right] \in \mathbb{R}^{2 n \times 2}$. As $S$ is symplectic, then $U_{1}^{J} U_{1}=I_{2}$ and the symplectic reflector allows us to set $U_{2}=\left[q_{2}, q_{n+2}\right] \in \mathbb{R}^{2 n \times 2}$. As $P_{1} S$ is still symplectic, $U_{2}$ verifies $U_{2}^{J} U_{2}=I_{2}$, and the symplectic reflector 
$P_{2}=\left(U_{2}+E_{2}\right)\left(I_{2}+E_{2}^{J} U_{2}\right)^{-1}\left(U_{2}+E_{2}\right)^{J}-I_{2 n}$ has the following form:

$$
P_{2}=\left(\begin{array}{cccc|cccc}
1 & 0 & \cdots & 0 & 0 & 0 & \cdots & 0 \\
0 & * & * & * & \vdots & * & * & * \\
\vdots & * & * & * & \vdots & * & * & * \\
0 & * & * & * & 0 & * & * & * \\
\hline 0 & \cdots & \cdots & 0 & 1 & 0 & \cdots & 0 \\
\vdots & * & * & * & 0 & * & * & * \\
\vdots & * & * & * & \vdots & * & * & * \\
0 & * & * & * & 0 & * & * & *
\end{array}\right)
$$

and verifies $P_{2} U_{2}=E_{2}$. As in step 1 , we obtain

$$
P_{2} P_{1} S=\left(\begin{array}{ccccc|cccccc}
1 & 0 & 0 & \ldots & 0 & 0 & 0 & 0 & \ldots & 0 \\
0 & 1 & 0 & \ldots & 0 & 0 & 0 & 0 & \ldots & 0 \\
0 & 0 & * & \ldots & * & 0 & 0 & * & \ldots & * \\
\vdots & \vdots & \vdots & \ddots & \vdots & \vdots & \vdots & \vdots & \vdots & \vdots \\
0 & 0 & * & \ldots & * & 0 & 0 & * & \ldots & * \\
\hline 0 & 0 & 0 & \ldots & 0 & 1 & 0 & 0 & \ldots & 0 \\
0 & 0 & 0 & \ldots & 0 & 0 & 1 & 0 & \ldots & 0 \\
0 & 0 & * & \ldots & * & 0 & 0 & * & \ldots & * \\
\vdots & \vdots & \vdots & \vdots & \vdots & \vdots & \vdots & \vdots & \ddots & \vdots \\
0 & 0 & * & \ldots & * & 0 & 0 & * & \ldots & *
\end{array}\right) .
$$

We thereby obtain $P_{n} \cdots P_{2} P_{1} S=I_{2 n}$, and then $S=S_{1} S_{2} \cdots S_{n}$ where $S_{k}=P_{k}^{J}$, which achieves the desired result.

Remark. In lemma 2.8, by using $U=[u-J u]$, where $u \in \mathbb{R}^{2 n}$ with $\|u\| \neq 0$, we obtain $S$ orthogonal and symplectic.

Lemma 2. Let $u \in \mathbb{R}^{2 s}$ be a nonzero 2 s-component real vector. The orthogonal symplectic reflector $S=\left(U+\sqrt{\alpha} E_{1}\right)\left(\alpha I_{2}+\right.$ $\left.\sqrt{\alpha} E_{1}^{J} U\right)^{-1}\left(U+\sqrt{\alpha} E_{1}\right)^{J}-I_{2 s}$, where $U=[u-J u]$ verifies $S u=\sqrt{\alpha} e_{1}$ with $\alpha=u^{T} u=\|u\|_{2}^{2}$.

Proof. As $U^{J} U=\alpha I_{2}$ with $\alpha=u^{T} u=\|u\|_{2}^{2}>0$, then a simple calculation gives the result.

\section{Symplectic SVD-like decomposition}

We describe here a new approach to compute a symplectic SVD-like decomposition for a $2 n$-by- $m$ rectangular real matrix $A$. It's based on a Schur form of skew-symmetric matrix $A^{T} J A$. We obtained the following result:

$$
S A Q=\left(\begin{array}{c|c|c|c}
\Sigma_{p} & 0 & 0 & 0 \\
0 & 0 & I_{q} & 0 \\
0 & 0 & 0 & 0 \\
\hline 0 & \Sigma_{p} & 0 & 0 \\
0 & 0 & 0 & 0
\end{array}\right)
$$

where $Q$ is an orthogonal matrix, $S$ is symplectic, and $\Sigma_{p}=\left\{\sigma_{1}, \cdots, \sigma_{p}\right\}, p=\frac{\operatorname{rank}\left(A^{T} J A\right)}{2}$.

Let $A$ be $2 n$-by- $m$ rectangular real matrix. We recall here an useful result, which is the Schur like form of the real skew-symmetric matrix $A^{T} J A[16,17]$. 
Theorem 1. Given a $2 n-b y-m$ real matrix $A$, there is a real orthogonal matrix $U$ such that

$$
A^{T} J A=U\left(\begin{array}{c|c|c}
0_{p} & \Sigma_{p}^{2} & 0 \\
-\Sigma_{p}^{2} & 0_{p} & 0 \\
0 & 0 & 0_{m-2 p}
\end{array}\right) U^{T}
$$

where $\Sigma_{p}=\operatorname{diag}\left(\sigma_{1}, \sigma_{2}, \ldots, \sigma_{p}\right), \sigma_{i}>0, \forall i$ and $2 p=\operatorname{rank}\left(A^{T} J A\right)$.

$\mathrm{Xu}[16,17]$ showed that for any $n$-by-2m real matrix $A$, there exists an orthogonal matrix $Q$ and a symplectic matrix $S$, such that $A=Q D S^{-1}$, where $D$ is in the following form,

$$
D=\left(\begin{array}{llllll}
\Sigma & 0 & 0 & 0 & 0 & 0 \\
0 & I & 0 & 0 & 0 & 0 \\
0 & 0 & 0 & \Sigma & 0 & 0 \\
0 & 0 & 0 & 0 & 0 & 0
\end{array}\right)
$$

where $\Sigma$ is positive diagonal. Symplectic $S V D$-like decomposition is effective for computing the structured canonical form of the Hamiltonian matrix $J A^{T} A$. Xu also proposed an algorithm for computing eigenvalues of $J A^{T} A$ using block $A_{11}$ and $A_{23}$ in step 1 of the algorithm (for more details, see section 2 in [17]). Although he obtained the eigenvalues, his algorithm does not compute the full decomposition of the poorly scaled matrices. We present a new constructive approach which is main result of this paper, in order to obtain the symplectic SVD-like decomposition and to compute the eigenvalues of Hamiltonian matrix $J A A^{T}$.

Theorem 2. ( Symplectic SVD-like decomposition) Let A be a $2 n-b y-m$ rectangular real matrix. For a symplectic real matrix $S \in \mathbb{R}^{2 n \times 2 n}$ and an orthogonal real matrix $Q \in \mathbb{R}^{m \times m}$,

$$
S A Q=\left(\begin{array}{c|c|c|c}
\Sigma_{p} & 0 & 0 & 0 \\
0 & 0 & I_{q} & 0 \\
0 & 0 & 0 & 0 \\
\hline 0 & \Sigma_{p} & 0 & 0 \\
0 & 0 & 0 & 0
\end{array}\right)
$$

Proof. Applying the real Schur decomposition to the skew-symmetric matrix $A^{T} J A$

$$
A^{T} J A=U\left(\begin{array}{c|c|c}
0_{p} & \Sigma_{p}^{2} & 0 \\
\hline-\Sigma_{p}^{2} & 0_{p} & 0 \\
\hline 0 & 0 & 0_{m-2 p}
\end{array}\right) U^{T}
$$

we construct $V_{k}=\frac{1}{\sigma_{k}} A U_{k} J_{2}^{T}$, where $U_{k}=U\left[\begin{array}{ll}e_{k} & e_{p+k}\end{array}\right]$ for $k=1,2, \cdots, p$. As we can easily verify $V_{k}^{J} V_{k}=I_{2}$, the $2 n$-by-2 matrix $V_{k}$ is symplectic. We have, $\mathscr{R}(A)=\operatorname{span}\{A U\}=\operatorname{span}\left\{A U^{[p]}\right\} \oplus \operatorname{span}\left\{A U^{[s]}\right\}$, where $U^{[p]}=U(:, 1: 2 p)$ and $U^{[s]}=U(:, 2 p+1: m)$. Therefore,

$$
\begin{aligned}
w \in \operatorname{span}\left\{A U^{[p]}\right\} & \Longrightarrow w=A U^{[p]} z, \text { where } z \in \mathbb{R}^{2 p} \\
& \Longrightarrow A^{T} J w=\left(A^{T} J A\right) U^{[p]} z \\
& \Longrightarrow A^{T} J w=U^{[p]}\left(\begin{array}{c|c|c}
0_{p} & \Sigma_{p}^{2} \\
\hline-\Sigma_{p}^{2} \mid 0_{p}
\end{array}\right) z
\end{aligned}
$$


and

$$
\begin{aligned}
w \in \operatorname{span}\left\{A U^{[s]}\right\} & \Longrightarrow w=A U^{[s]} y, \text { where } y \in \mathbb{R}^{m-2 p} \\
& \Longrightarrow A^{T} J w=\left(A^{T} J A\right) U^{[s]} y=0_{\mathbb{R}^{m}}
\end{aligned}
$$

Thus,

$$
\begin{gathered}
w \in \operatorname{span}\left\{A U^{[p]}\right\} \cap \operatorname{span}\left\{A U^{[s]}\right\} \\
\Longrightarrow\left\{\begin{array}{l}
w=A U^{[p]} z, \text { where } z \in \mathbb{R}^{2 p} \\
A^{T} J w=U^{[p]}\left(\frac{0_{p} \mid \Sigma_{p}^{2}}{-\Sigma_{p}^{2} \mid 0_{p}}\right) z=0_{\mathbb{R}^{m}}
\end{array}\right. \\
\Longrightarrow z=0_{\mathbb{R}^{2 p} \text { and then } w=0_{\mathbb{R}^{2 n}} .}
\end{gathered}
$$

This proves $\operatorname{span}\left\{A U^{[p]}\right\} \cap \operatorname{span}\left\{A U^{[s]}\right\}=\left\{0_{\mathbb{R}^{2 n}}\right\}$. As a consequence, we have $\operatorname{dim}\left(\operatorname{span}\left\{A U^{[p]}\right\}\right)=2 p$ and $\operatorname{dim}\left(\operatorname{span}\left\{A U^{[s]}\right\}\right)=q=r-2 p$, where $r$ is the $\operatorname{rank}$ of $A$. The null space $\mathscr{N}(A)$ of the matrix $A$ verifies $\mathscr{N}(A) \subset \operatorname{span}\left\{U^{[s]}\right\}$.

Suppose that $\mathscr{N}(A)=\operatorname{span}\left\{U^{[s]}(:, q+1: m-2 p)\right\}=\operatorname{span}\{U(:, 2 p+q+1: m)\}$. We then set $v_{j}=A u_{j}$ for $j=2 p+1, \cdots, r=2 p+q$. As $v_{j}=A u_{j}$ where $u_{j} \in \mathscr{N}\left(A^{T} J A\right)$, then $\left(v_{j}\right)_{2 p+1 \leq j \leq r}$ are $J$-orthogonal and $V_{k}^{T} J v_{j}=0$ for all $1 \leq k \leq p$ and $2 p+1 \leq j \leq r$. Let us construct the $2 n$-by- $r$ matrix $V$ as follow: $V=\sum_{k=1}^{p} V_{k}\left[e_{k} e_{p+k}\right]^{T}+\sum_{j=2 p+1}^{r} v_{j} e_{j}^{T}$ where $e_{i}$ is the $i^{\text {th }}$ canonical basis vector of $\mathbb{R}^{r}$. We have $V^{T} J_{2 n} V=\left(\begin{array}{ccc}0_{p} & I_{p} & 0 \\ -I_{p} & 0_{p} & 0 \\ 0 & 0 & 0_{q}\end{array}\right)$. And let's the $m$-by- $m$ orthogonal matrix $Q$ given by $Q=\sum_{k=1}^{p} U_{k} J_{2}^{T}\left[\begin{array}{ll}e_{k} & e_{p+k}\end{array}\right]^{T}+\sum_{j=2 p+1}^{m} u_{j} e_{j}^{T}$, here $e_{i}$ is the $i^{t h}$ canonical basis vector of $\mathbb{R}^{m}$. We have

$$
A Q=V\left(\begin{array}{c|c|c}
\Sigma_{p} & 0 & 0 \\
\hline 0 & \Sigma_{p} & 0 \\
\hline 0 & 0 & I_{q \times(m-2 p)}
\end{array}\right) .
$$

Step 1: As $V_{1}=\left[v_{1}, v_{p+1}\right] \in \mathbb{R}^{2 n \times 2}$ verifies $V_{1}^{J} V_{1}=I_{2}$, then the symplectic reflector $S_{1}=\left(V_{1}+E_{1}\right)\left(I_{2}+E_{1}^{J} V_{1}\right)^{-1}\left(V_{1}+\right.$ $\left.E_{1}\right)^{J}-I_{2 n}$ verifies $S_{1} V_{1}=E_{1}$. From the $J$-orthogonality given above, we have $\left(S_{1} V_{1}\right)^{T} J\left(S_{1} v_{j}\right)=E_{1}^{T} J\left(S_{1} v_{j}\right)=0$ for all $j \neq 1$ and $j \neq p+1$. Thus, the $1^{s t}$ and the $(n+1)^{t h}$ components of $\left(S_{1} v_{j}\right)$ are equal to zero. We obtain

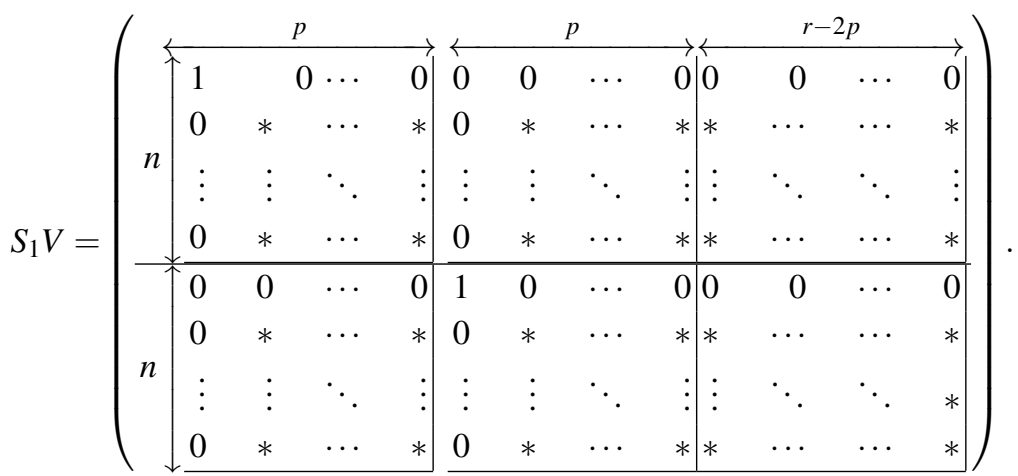

We can therefore update the value of $V: V \longleftarrow S_{1} V$.

Step 2: $\quad V_{2}=\left[v_{2}, v_{p+2}\right] \in \mathbb{R}^{2 n \times 2}$ again verifies $V_{2}^{J} V_{2}=I_{2}$. Then, the symplectic reflector $S_{2}=\left(V_{2}+E_{2}\right)\left(I_{2}+E_{2}^{J} V_{2}\right)^{-1}\left(V_{2}+E_{2}\right)^{J}-I_{2 n}$ is such that $S_{2} V_{2}=E_{2}$. Again, from the $J$-orthogonality, we have $E_{2}^{T} J\left(S_{2} v_{j}\right)=0$ for all $j \neq 2$ and $j \neq p+2$. Thus, the $2^{\text {nd }}$ and the $(n+2)^{t h}$ components of $\left(S_{2} v_{j}\right)$ are equal to zero. We 
obtain

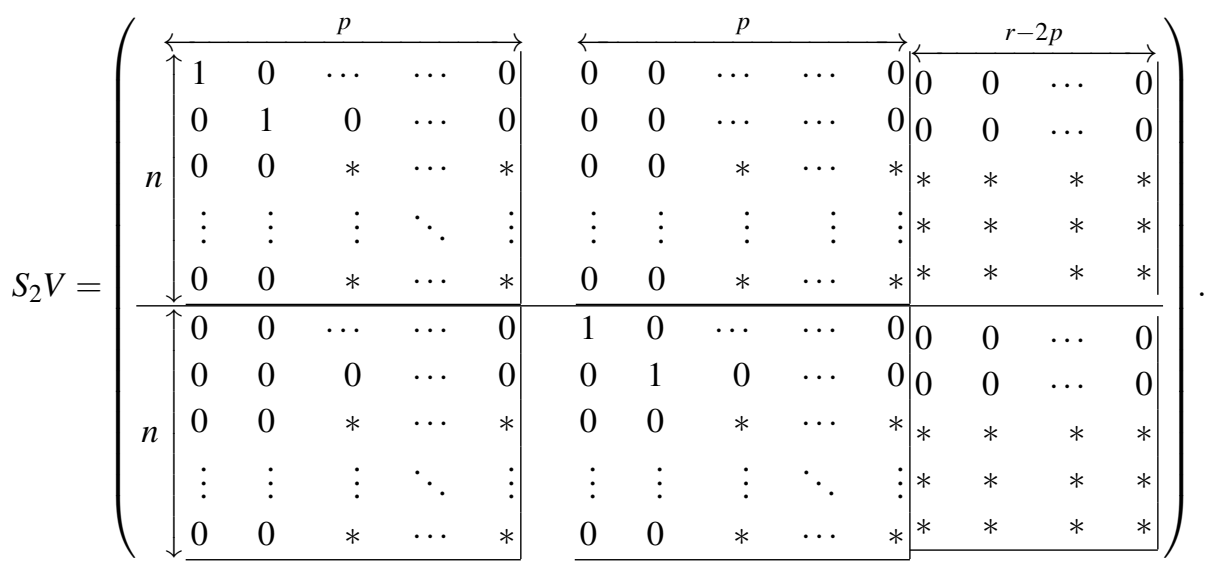

Continuing until the $p^{\text {th }}$ step, we obtain

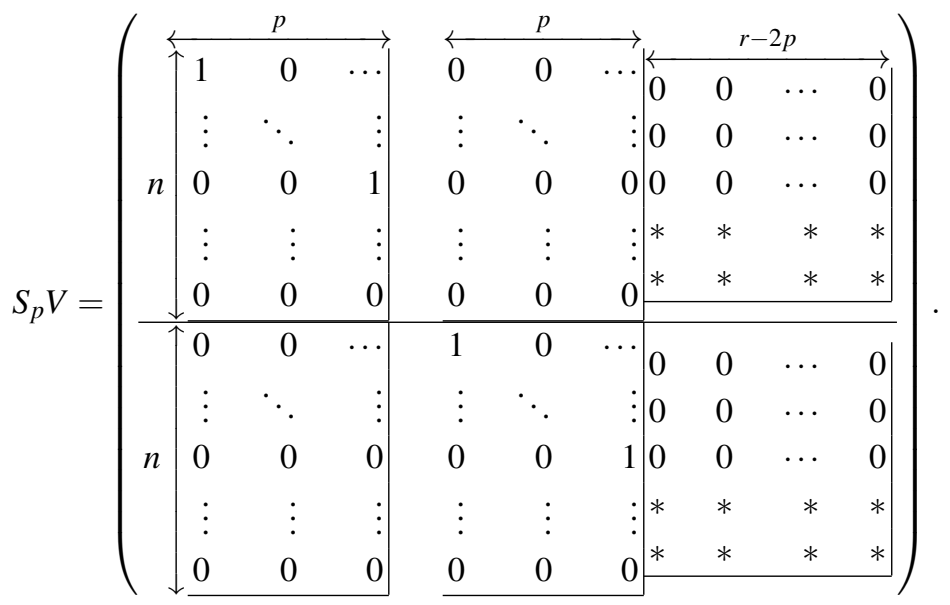

We can therefore update the value of $V: V \longleftarrow S_{p} V$. As $\operatorname{rank}(V)=\operatorname{rank}(A)=r=2 p+q$ and according to the lemma above, there exist $q$ reflectors $S_{p+1}, S_{p+2}, \cdots, S_{p+q}$ such that $V \longleftarrow S_{p+q}\left(\cdots S_{p+2}\left(S_{p+1} V\right)\right)$ is reduce as follows:

$$
V=\left(\begin{array}{cccc} 
& & & 0_{p \times q} \\
I_{n \times p} & 0_{n \times p} & R \\
& & 0_{(n-(p+q)) \times q} \\
0_{n \times p} & I_{n \times p} & & 0_{n \times q}
\end{array}\right)
$$

where $R \in \mathbb{R}^{(r-2 p) \times(r-2 p)}$ is nonsingular triangular matrix. By setting

$$
S_{p+q+1}=\operatorname{diag}\left(I_{p}, R^{-1}, I_{(n-(p+q))}, I_{p}, R, I_{(n-(p+q))}\right)
$$

which is a symplectic matrix, $V \longleftarrow S_{p+q+1} V$ is finally in the following form:

$$
V=\left(\begin{array}{cccc} 
& & & 0_{p \times q} \\
I_{n \times p} & 0_{n \times p} & I_{q} \\
& & 0_{(n-(p+q)) \times q} \\
0_{n \times p} & I_{n \times p} & & 0_{n \times q}
\end{array}\right) .
$$


Let $S=S_{p+q+1} S_{p+q} \cdots S_{2} S_{1}$. We then have

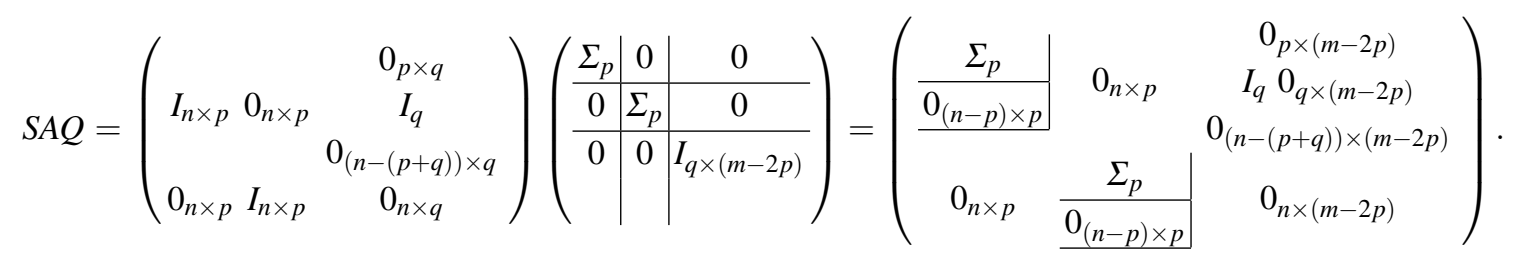

which corresponds to the hypothesized form.

\section{Algorithm ( Symplectic SVD-like algorithm)}

Input : Matrix $A \in \mathbb{R}^{2 n \times m}$.

Output : A symplectic matrix $S \in \mathbb{R}^{2 n \times 2 n}$, an orthogonal matrix $Q \in \mathbb{R}^{m \times m}$ and the desired SVD-like decomposition.

1. Compute a canonical Schur form of skew-symmetric matrix $M=A^{T} J A \in \mathbb{R}^{m \times m}$ such that

$$
U M U^{T}=\left(\begin{array}{c|c|c}
0_{p} & \Sigma_{p}^{2} & 0 \\
\hline-\Sigma_{p}^{2} & 0_{p} & 0 \\
\hline 0 & 0 & 0_{m-2 p}
\end{array}\right) .
$$

2. For $k=1, \cdots, p$, where $2 p=\operatorname{rank}(M)$

Compute $V_{k}=\frac{1}{\sigma_{k}} A U_{k} J_{2}^{T}$ where $U_{k}=U\left[e_{k} e_{p+k}\right]$.

\section{End For}

3. Set $v_{j}=A u_{j}$ for $j=2 p+1, \cdots, r$ with $r=\operatorname{rank}(A)=2 p+q$.

4. Set $V=\sum_{k=1}^{p} V_{k}\left[e_{k} e_{p+k}\right]^{T}+\sum_{k=2 p+1}^{r} v_{j} e_{j}^{T}$

and $Q=\sum_{k=1}^{p} U_{k} J_{2}^{T}\left[e_{k} e_{p+k}\right]^{T}+\sum_{k=2 p+1}^{m} u_{j} e_{j}^{T}$.

5. Set $S=I_{2 n}$ and

For $k=1, \cdots, p$

Compute a symplectic reflector $S_{k}$ associated to $V_{k}$

Update $V \leftarrow S_{k} V$ and $S \leftarrow S S_{k}$.

End For

6. For $k=1, \cdots, q=r-2 p$

Using lemma 2.2, we can compute a symplectic reflector $S_{k}$ associated with $v_{k}$.

Update $V \leftarrow S_{k} V$ with

$$
S_{k} V=\left(\begin{array}{ccc} 
& & 0_{p \times q} \\
I_{n \times p} & 0_{n \times p} & R \\
& & 0_{(n-(p+q)) \times q} \\
0_{n \times p} & I_{n \times p} & 0_{n \times q}
\end{array}\right)
$$

and $S \leftarrow S S_{k}$.

\section{End For}

7. Updating $V \leftarrow S_{p+q+1} V$ and $S \leftarrow S S_{p+q+1}$

where $S_{p+q+1}=\operatorname{diag}\left(I_{p}, R^{-1}, I_{(n-(p+q))}, I_{p}, R, I_{(n-(p+q))}\right)$.

with $R=V(p+1: p+q, 2 p+1: r)$ 
8. $S A Q=\Sigma=\left(\begin{array}{c|c|c|c}\Sigma_{p} & 0 & 0 & 0 \\ 0 & 0 & I_{q} & 0 \\ 0 & 0 & 0 & 0 \\ \hline 0 & \Sigma_{p} & 0 & 0 \\ 0 & 0 & 0 & 0\end{array}\right)$.

Computation of the symplectic SVD-like decomposition of a $2 n$-by- $m$ matrix $A$ can be viewed as solving an eigenvalue problem of Hamiltonain $J A A^{T}$ without having to compute the product of the full matrix, as we shown in the first example below. Let $A$ be a $2 n$-by- $m$ rectangular matrix. Using algorithm 3.1, we obtain the following result:

$$
S A Q=\Sigma=\left(\begin{array}{c|c|c|c}
\Sigma_{p} & 0 & 0 & 0 \\
0 & 0 & I_{q} & 0 \\
0 & 0 & 0 & 0 \\
\hline 0 & \Sigma_{p} & 0 & 0 \\
0 & 0 & 0 & 0
\end{array}\right) .
$$

Therefore,

$$
J A A^{T}=\Sigma=S^{J}\left(\begin{array}{ccc|ccc}
0 & 0 & 0 & \Sigma_{p}^{2} & 0 & 0 \\
q w<0 & 0 & 0 & 0 & 0 & 0 \\
0 & 0 & 0 & 0 & 0 & 0 \\
\hline-\Sigma_{p}^{2} & 0 & 0 & 0 & 0 & 0 \\
0 & -I_{q} & 0 & 0 & 0 & 0 \\
0 & 0 & 0 & 0 & 0 & 0
\end{array}\right) S
$$

which is the structured canonical form of Hamiltonian matrix $J A A^{T}$.

\section{Numerical examples}

We show here the results of numerical tests to compare the method shown in algorithm 3.1 with that of Xu [17]. We computed the eigenvalues of Hamiltonian matrix $J A A^{T}$ and calculated the error of symplectic SVD-like decomposition. these experiments were carried out with Matlab 7.8.0 (R2009a) and run on a Core Duo Pentium processor.

Let $A$ be a rectangular matrix, defined as follows:

$$
A=Q\left(\begin{array}{ccc|ccc}
\Sigma & 0 & 0 & 0 & 0 & 0 \\
\hline 0 & I & 0 & 0 & 0 & 0 \\
\hline 0 & 0 & 0 & \Sigma & 0 & 0 \\
\hline 0 & 0 & 0 & 0 & 0 & 0
\end{array}\right) U^{T}
$$

where $Q$ is a random orthogonal matrix and $U$ is a $14 \times 14$ random orthogonal symplectic matrix. We calculated the error that occurred when computing the symplectic SVD-like decomposition and compared the relative errors in the computed eigenvalues for Hamiltonian matrix $J A A^{T}$ obtained by the proposed method and that of $\mathrm{Xu}$ [17].

- $\Sigma=\operatorname{diag}(4,3,2,1)$, the error in computing symplectic SVD-like decomposition was $1.1743 e-014$ with our method and $1.1897 e-014$ with that of $\mathrm{Xu}$. The relative errors for nonzero eigenvalues with the two methods are shown in the table below:

\begin{tabular}{|c|c|c|}
\hline eigenvalue & Algorithm3.1 & $\mathrm{Xu}[17]$ \\
\hline $\pm 16 i$ & $2.2204 e-015$ & $2.8 e-014$ \\
\hline $\pm 9 i$ & $1.1842 e-015$ & $5.3 e-015$ \\
\hline $\pm 4 i$ & $2.2204 e-015$ & $1.2 e-013$ \\
\hline $\pm i$ & $1.3323 e-015$ & $6.9 e-015$ \\
\hline
\end{tabular}


- $\Sigma=\operatorname{diag}\left(10^{-2}, 10^{-1}, 1,10^{2}\right)$, the error in computing symplectic SVD-like decomposition was $4.3215 e-009$ with our method and 148.4 with that of $\mathrm{Xu}$. As in the previous example, although the eigenvalues are computed correctly by Xu's method, computation of the symplectic SVD-like decomposition was incomplete. The relative errors for nonzero eigenvalues with the two methods are shown in the table bellow:

\begin{tabular}{|c|c|c|}
\hline eigenvalue & Algorithm3.1 & $\mathrm{Xu}[17]$ \\
\hline $\pm 10^{4} i$ & $1.5421 e-015$ & $6.4681 e-015$ \\
\hline $\pm i$ & $3.2846 e-014$ & $6.4567 e-014$ \\
\hline $\pm 10^{-2} i$ & $5.4357 e-012$ & $8.5701 e-012$ \\
\hline $\pm 10^{-4} i$ & $3.5356 e-009$ & $5.1430 e-009$ \\
\hline
\end{tabular}

- $\Sigma=\operatorname{diag}\left(10^{-4}, 10^{-2}, 1,10^{2}\right)$, the error in computing symplectic SVD-like decomposition was $1.9411 e-008$ with our method and 172.4 with that of $\mathrm{Xu}$. As in the previous example, although the eigenvalues are computed correctly by Xu's method, computation of the symplectic SVD-like decomposition was incomplete. The relative errors for nonzero eigenvalues with the two methods are shown in the table below:

\begin{tabular}{|c|c|c|}
\hline eigenvalue & Alg3.1 & Xu method[17] \\
\hline $\pm 10^{4} i$ & $1.0914 e-015$ & $9.0541 e-015$ \\
\hline $\pm i$ & $1.2679 e-013$ & $1.3 e-015$ \\
\hline $\pm 10^{-4} i$ & $1.5679 e-009$ & $5.7 e-012$ \\
\hline $\pm 10^{-8} i$ & $1.6254 e-006$ & $4.3456 e-010$ \\
\hline
\end{tabular}

In this example, the relative error of the computed eigenvalue corresponding to $\lambda=10^{-8}$ given by matlab was 12.6377 and the absolute error was $1.2638 e-007$.

\section{Conclusion}

We have presented a numerical method for computing symplectic SVD-like decomposition. It is based on the canonical Schur-form of skew-symmetric matrix, as used by Xu [16,17]. The canonical form of the skew-symmetric matrix $A J A^{T}$, the Hamiltonian matrix $J A^{T} A$ and the skew-Hamiltonian matrix $A^{J} A$ can be derived from such a decomposition. The numerical examples presented show the effectiveness of proposed algorithm.

\section{References}

[1] S. Agoujil, Nouvelles méthodes de factorisation pour des matrices structurées, PHD Thesis. Faculté des Sciences et TechniquesMarrakech. Département de Mathématiques et Informatique (February 2008).

[2] S. Agoujil and A. H. Bentbib, On the reduction of Hamilotonian matrices to a Hamiltonian Jordan canonical form, Int. Jour. Math. Stat. (IJMS) 4, 12-37, Spring (2009).

[3] S. Agoujil and A. H. Bentbib, New symplectic transformation on $\mathbb{C}^{2 n \times 2}$ : Symplectic reflectors, Int. Jour. of Tomography and Statistics (IJTS) 11, 99-117, Summer (2009).

[4] A.G. Akritas, G.I. Malaschinok and P.S. Vigglas, The SVD-Fundamental Theorem of Linear Algebra, Non Linear Analysis Modelling and Control,Vol 11, 123-136, (2006).

[5] M. Bassour and A. H. Bentbib, Factorization of $R^{J} R$ of skew-Hamiltonian matrix using its Hamiltonian square root, Int. Jour. of Tomography and Statistics (IJTS) 8 Springer(2011).

[6] A. H. Bentbib and A. Kanber, A method for solving Hamiltonian eigenvalue problem, Int. Jour. Math. Stat. (IJMS) 7 Winter(2010).

[7] C. Brezinski, Computational Aspects of Linear Control Numerical Methods and Algorithms. Springer, (2002).

[8] G. Golub, W. Kahan, Calculating the Singular Values and Pseudo-Inverse of Matrix, J. SIAM Numerical Analysis, Ser. B, Vol 2 N. 2 printed in U. S. A, 205-224, (1965). 
[9] G. Golub and C. Reinsch, Singular Value Decomposition and Least Square Solutions, In J. H. Wilkinson and C. Reinsch, editors, Linear Algebra, volume II of Handbook for Automatic Computations, chapter I/10, 34-151. Springer Verlag, (1971).

[10] R.J. Duffin, The Rayleigh-Ritz method for dissipative or gyroscopic systems, Quart. Appl. Math., 18, 215-221, (1960).

[11] P. Lancaster, Lambda-Matrices and Vibrating Systems, Pergamon Press, Oxford, UK, (1966).

[12] F. Tisseur and K. Meerbergen, The quadratic eigenvalue problem, SIAM Review, 43, 235-286, (2001).

[13] N. E. Mastorakis, Positive singular Value Decomposition, Recent Advances in Signal Processing and Communication (dedicated to the father of Fuzzy Logic, L. Zadeh), WSEAS-Press, pp:7-17, (1999).

[14] N. E. Mastorakis, The singular Value Decomposition (SVD) in Tensors (Multidimensional Arrays) as an Optimization Problem. Solution via Genetic Algorithms and method of Nelder-Mead, Proceeding of the 6th WSEAS Int. Conf. on System Theory Scientific Computation, Elounda, Greece, August 21-23, pp: 7-13, (2006).

[15] V.Mehrmann, The Autonomus Linear Quadratic Control Problem, Theory and Numerical Solution, Number 163 in Lecture Notes in Control and Information Sciences. Springer- Verlag, Heidelberg, July (1991).

[16] H. Xu, An SVD-like matrix decomposition and its applications, Linear Algebra and its Applications. 368, 1-24, (2003).

[17] H. Xu, A Numerical Method For Comuping An SVD-like matrix decomposition, SIAM journal on matrix analysis and applications. 26, 1058-1082, (2005).

[18] C. Van Loan, A Symplectic method for approximating all the eigenvalues of Hamiltonian matrix Linear Alg. Appl. 61, 233-251, (1984). 